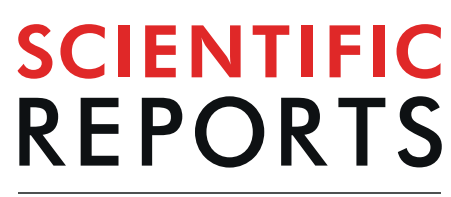

natureresearch

Check for updates

\title{
Comparative and Phylogenetic Analyses of the Complete Chloroplast Genomes of Six Almond Species (Prunus spp. L.)
}

\author{
Wei Wang ${ }^{1}$, Tao Yang ${ }^{2}$, Hui-Ling Wang ${ }^{3}$, Zhen-Jian $\mathrm{Li}^{1}$, Jian-Wei Ni ${ }^{1}$, Shang Su ${ }^{1}$ \& \\ Xin-Qiao Xü
}

As a source of genetic variation, almond germplasm resources are of great significance in breeding. To better reveal the mutation characteristics and evolution patterns of the almond chloroplast (ср) genome, the complete cp genomes from six almond species were analyzed. The lengths of the chloroplast genome of the six almond species ranged from 157,783 bp to 158,073 bp. For repeat sequence analysis, 53 pairs of repeats ( $30 \mathrm{bp}$ or longer) were identified. A total of $117 \mathrm{SSR}$ loci were observed, including 96 polymorphic SSR loci. Nine highly variable regions with a nucleotide variability (Pi) higher than 0.08, including rps16, rps16-psbK, atpF-atpH, rpoB, ycf3-rps4, rps4-ndhJ, accD-psal and $r p s 7-o r f 42$ (two highly variable regions) were located. Based on the chloroplast genome evolution analysis, three species $(P$. tenella, $P$. pedunculata and $P$. triloba) and wild cherry $(P$. tomentosa) were grouped into clade I. Clade II consisted of two species ( $P$. mongolica and $P$. tangutica) and wild peach ( $P$. davidiana). Clade III included the common almond ( $P$. dulcis), cultivated peach ( $P$. persica) and GanSu peach ( $P$. kansuensis). This result expands the researchers' vision of almond plant diversity and promotes an understanding of the evolutionary relationship among almond species. In brief, this study provides abundant resources for the study of the almond chloroplast genome, and has an important reference value for study of the evolution and species identification of almond.

The common almond (Prunus dulcis (Mill.) D.A.Webb), a member of the family Rosaceae and order Rosales, is widely and mainly distributed in the temperate zones of Europe, America, and Asia ${ }^{1,2}$. Among the different varieties of tree nuts, almonds are the second most consumed worldwide, behind peanuts. In 2018, the volume of almonds consumed worldwide amounted to 1.19 million metric tons ${ }^{1}$. In addition to common almonds, there are more than thirty almond species in the world, including partially cultivated and wild varieties ${ }^{2}$. Five wild almond resources have been reported in China, including the diploid species wild almond (Prunus tenella Batsch), Mongolic almond (Prunus mongolica Maxim.), and Tangut almond (Prunus tangutica (Batalin) Koehne), the octoploid species, flowering almond (Prunus triloba Lindl.), and the dodecaploid species, longstalk almond (Prunus pedunculata (Pall.) Maxim.) ${ }^{3-6}$. All the almond species are highly adapted to a cold and dry climate, which may indicate an important gene pool. Therefore, it is of great significance to identify the almond genetic resources and evaluate their industrial application value for effective utilization and preservation.

Wild almond species usually grow in areas of altitudes between $1,100 \mathrm{~m}$ and 2,700 $\mathrm{m}$ and at latitudes between 28 and $38 \mathrm{~N}$ and longitudes between 41 and $54 \mathrm{E}^{7,8}$. It is reported that there are five wild species in China, indicating that China is one of the central origins of almonds ${ }^{4,5,7}$. Based on taxonomy, wild almond species and the common almond were classified as a separate genus, Amygdalus L. ${ }^{9}$, outside of the genus Prunus L. Subsequently, Gorttapeh also classified the almond resources in the subgenus Amygdalus $\mathrm{L}^{2,10}$ as a subgenus of Prunus. At present, most authors have suggested putting almond species in the Prunus genus rather than considering them as an independent genus ${ }^{8,11}$. Polyploidy exists in the six species, and it is not easy to distinguish between them by evolutionary analysis based on genome sequence and phenotype, which leads to the unclear evolutionary relationships

${ }^{1}$ Key Laboratory of Silviculture of the State Forestry Administration, The Institute of Forestry, The Chinese Academy of Forestry, Yi HeYuan Hou, Beijing, 100091, China. ${ }^{2}$ Shaanxi Academy of Forestry, Shaanxi, 719000, China. ${ }^{3}$ Institute of Forestry and Pomology, Beijing Academy of Agriculture and Forestry Sciences, Beijing, 100097, China. ${ }^{凶}$-mail: greatkingww@163.com 


\begin{tabular}{|l|l|l|l|l|}
\hline Species & Origin & Collection site & Latitude & Longitude \\
\hline $\begin{array}{l}\text { P. dulcis var. } \\
\text { ZHIPI }\end{array}$ & Cultivar & $\begin{array}{l}\text { Kashi, Xinjiang, } \\
\text { China }\end{array}$ & 38.22 & 77.18 \\
\hline P. tangutica & Wild & Aba, Sichuan, China & 32.3 & 103.38 \\
\hline P. pedunculata & Wild & Yulin, Shanxi, China & 38.51 & 109.52 \\
\hline P. mongolica & Wild & $\begin{array}{l}\text { Alashan, Neimenggu, } \\
\text { China }\end{array}$ & 39.46 & 105.3 \\
\hline P. tenella & Wild & $\begin{array}{l}\text { Aertai, Xinjiang, } \\
\text { China }\end{array}$ & 48.18 & 86.34 \\
\hline P. triloba & Wild & $\begin{array}{l}\text { Chicheng, Hebei, } \\
\text { China }\end{array}$ & 41.11 & 116.04 \\
\hline
\end{tabular}

Table 1. Description of the six almond species.

of the wild almond resources ${ }^{3-6}$. However, there is no polyploidy in the chloroplast (cp) genome. In addition, compared with nuclear and mitochondrial genomes, the cp sequence conservation makes it a more common and effective tool for plant phylogenomic analysis.

The chloroplast organelle is the location for photosynthesis and carbon fixation in plants. Because of its unique maternal inheritance and low silent nucleotide substitution rate, chloroplast DNA (cpDNA) has become a useful tool for the study of plant genetic evolution and for interspecific and intraspecific polymorphism identification $^{12-15}$. In addition, the chloroplast is not polyploid, which can be utilized for the genetic analysis of polyploid plants. With the introduction of new sequencing technology, a large amount of DNA sequence data can be obtained at a relatively low cost. The acquisition of DNA sequence data is beneficial for exploring plant evolution and formulating breeding strategies at the molecular level. Because the chloroplast genome evolves slowly relative to the nuclear genome, chloroplast sequences provide valuable resources for studying population genetics, phylogeography, phylogeny, and species identification.

The chloroplast genome is a relatively highly conserved circular DNA with a size ranging from 115 to 165 $\mathrm{kb}^{12-15}$. Generally, the cpDNA genome has two large reverse repeat (IR) copies separated by large single-copy (LSC) and small single-copy (SSC) regions. Chloroplast genomes usually contain 110-130 different genes, which have highly conserved gene sequences. Most of them ( 79) encode proteins mainly involved in photosynthesis, while other genes encode approximately thirty transfer RNAs (tRNA) and 4 ribosomal RNAs (rRNA) ${ }^{6,12}$. The cp genome comparative analysis not only provides information on the genome structure but also plays a significant role in understanding the cp genome evolution, phylogeny and species identification ${ }^{13-15}$.

Here, we present the complete and annotated DNA sequences of the cp genomes of five almond resources. Our research purposes were as follows: (1) to study the overall structure of the cp genomes; (2) to detect the variations in the repeat sequences and the simple sequence repeats (SSRs) in the six almond cp genomes; (3) to screen rapidly evolving DNA regions in the six chloroplast genomes; and (4) to analyze the phylogenetic relationship using the sequence data of the chloroplast genome. These results will supply rich molecular tools for further phylogenetic analysis, population genetics analysis, and species identification and will contribute to almond breeding.

\section{Materials and methods}

Chloroplast DNA sequencing and genome assembly. Fresh leaves of the almond species were collected from a single plant of each species in different distribution areas (Table 1 ). Approximately $5 \mathrm{~g}$ fresh leaves were harvested as outlined by the improved extraction method ${ }^{16}$ for chloroplast DNA separation. The chloroplast DNA was extracted using the high-salt saline plus Percoll gradient method. After DNA separation, $1 \mu \mathrm{g}$ of purified DNA was segmented and used to construct a short insertion library (insert size $430 \mathrm{bp}$ ) according to the manufacturer's instructions (Illumina, San Diego, CA, USA) ${ }^{17}$, followed by sequencing on the Illumina HiSeq. 4000.

Before assembly, the raw reads were filtered. This filtering step was performed to delete reads with adaptors, reads with a display quality score of less than $20(\mathrm{Q}<20)$, reads that contain a percentage of an uncalled base ("N" character) equal to or greater than $10 \%$, and repeat sequences. The reconstruction of the chloroplast genome was based on a combination of de novo and reference-guided assemblies. The following three steps were used to assemble the $\mathrm{cp}$ genome ${ }^{18}$. First, SOAPdenovo2.04 was used to assemble the filtered reads into contigs ${ }^{19}$. Next, BLAST was used to compare contigs with the reference genome of the six almond species and to align contigs (the similarity and query coverage were more than $80 \%$ ) according to the comparison genomes. Finally, clean reads were packaged into the assembled draft cp genome to correct the wrong bases, and most of the gaps were filled by the local assembly.

Genome annotation. The chloroplast genes were annotated with an online DOGMA tool ${ }^{20}$, using the default parameters to predict ribosomal RNA (rRNA) genes, transfer RNA (tRNA) genes, and protein-coding genes. A whole chloroplast genome BLAST search (minimal alignment length percentage $>=40 \%$, E-value $<=$ $1 \times 10^{-5}$ ) was performed against five databases ${ }^{21}$ GO $^{22}$ (Gene Ontology), Swiss-Prot ${ }^{23}$, NR (Non-Redundant Protein Database), COG (Clusters of Orthologous Groups) ${ }^{24,25}$, and KEGG (Kyoto Encyclopedia of Genes and Genomes $)^{26-28}$. The chloroplast genome maps of the six almond species were assembled by using OrganellarGenomeDRAW v1.2 $2^{29}$.

Repeat sequences and SSRs characteristics. Using REPuter ${ }^{30}$, the size and location of direct (forward), reverse (palindrome), complement and reverse repeat sequences in the chloroplast genomes of the six almond 


\begin{tabular}{|l|l|l|l|l|l|l|}
\hline & P. dulcis & P. pedunculata & P. triloba & P. mongolica & P. tangutica & P. tenella \\
\hline GeneBank Number & MH727486 & MG869261 & MH748555 & MH727485 & MH744156 & MH727487 \\
\hline Total Sequence Length & 157,783 & 157,873 & 157,816 & 158,074 & 158,049 & 158,066 \\
\hline Large Single Copy (LSC) & 85,921 & 86,074 & 86,021 & 86,316 & 86,226 & 86,260 \\
\hline Small Single Copy (SSC) & 19,100 & 19,029 & 19,023 & 18,992 & 19,063 & 19,056 \\
\hline Inverted Repeat Region (IR) & 26,380 & 26,384 & 26,385 & 26,382 & 26,379 & 26,374 \\
\hline Total Number of Gene & 136 & 136 & 136 & 136 & 136 & 136 \\
\hline Protein-Coding Genes & 91 & 91 & 91 & 91 & 91 & 91 \\
\hline tRNA & 37 & 37 & 37 & 37 & 37 & 37 \\
\hline rRNA & 8 & 8 & 8 & 8 & 8 & 8 \\
\hline GC\% & 36.77 & 36.78 & 36.79 & 36.76 & 36.76 & 36.73 \\
\hline Reference & This article & Wang et al. (2019) & This article & This article & This article & This article \\
\hline
\end{tabular}

Table 2. Comparison of the complete chloroplast genome contents of the six almond species.

species were identified. For all the repeat types, the hamming distance, equal to the greater sequence identity, in REPuter was equal to 3, or the constraint set was $90 \%$. Using MISA Perl script ${ }^{31}$, simple sequence repeats (SSRs) were detected with thresholds of 10 repeat units for mononucleotide SSRs, six repeat units for dinucleotide SSRs, and five repeat units for tri-, tetra-, penta-, and hexa-nucleotide SSRs.

Identification of high variable regions. In DnaSP v5.10, based on the alignment sequence matrix of the cp genomes, the sliding window analysis method was used to evaluate the nucleotide variability (Pi) and polymorphic sites (S) with a 200 bp step length and a 600 bp window length ${ }^{32}$. Highly variable regions were defined as the number of polymorphic loci greater than the sum of the average and double the standard deviation. The annotated cp genome determined the locations of these highly variable regions. DnaSP v5.10 was used to evaluate the variable sites, parsimony-informative sites and nucleotide diversity of the hypervariable regions.

Phylogenomic analysis. To reveal the evolutionary relationship among the six almond species of the Rosaceae class, a total of 33 complete cp genomes of the family Rosaceae were collected from GenBank, with Vitis Vinifera and Syzygium cumini as the outer group (Table S1). Using PAUP v4 and 1000 random addition sequences used for a Heuristic search, the phylogenetic trees that were based on maximum parsimony analysis were constructed ${ }^{33}$. The phylogenetic trees based on maximum likelihood analysis were constructed by RAxML-win32-100315, and the bootstrap repetition rate was $1000^{34}$. The phylogenetic trees based on Bayesian inference was established by MrBayes v3.2.4 $4^{35}$. The $\mathrm{cp}$ genome sequences were compared and visualized by MAFFT v $7.149^{36}$.

\section{Results and Discussion}

Genome sequencing and assembly. Among the six almonds species sequenced in this research, $2874 \mathrm{Mb}$ to $4311 \mathrm{Mb}$ of raw data was generated on the Illumina sequencing system, with an average read length of $150 \mathrm{bp}$. From $2679 \mathrm{Mb}$ to $3995 \mathrm{Mb}$ of reads, the complete chloroplast genome sequence with $16.95 \times$ to $25.31 \times$ coverage was extracted. Five new almond cp genome sequences were submitted to GenBank (Table 2). The five almond cp genome lengths ranged from 157,783 bp (P. dulcis var. ZHIPI) to 158,065 bp (P.tenella; Fig. 1, Table 2).

Comparative analysis of the six almond chloroplast genomes. The six almond cp genomes composed of circular double-stranded DNA with a quadripartite structure, consist of an LSC region of 85,921 bp-86,316 bp, an SSC region of 18,992 bp-19,100 bp, and an IR region of 26,374 bp-26,386 bp. The overall GC content of the six almond species was $36.73 \%-36.79 \%$ (Table 2), with a low GC content and a high AT content. Many angiosperm cp genomes have been indicated to be characterized by a low GC content and a high AT content $\mathrm{t}^{12,13,37}$.

A total of 136 coding genes (Fig. 1, Table 2) were identified, including 91 protein-coding genes, 37 tRNA genes, and eight rRNA genes. Among them, 19 duplicate genes, seven protein-coding genes, eight tRNA genes, and four rRNA genes were found in the IR region. The coding genes of the six almond cp genomes were annotated in the same order.

Among the 136 genes, 21 have one intron (13 protein-coding genes and 8 tRNA genes), and two genes ( $c l p P$ and $y c f 3$ ) have two introns (both protein-coding) (Table $3 \&$ S2, Figure S1). Of the 21 genes with introns, 12 are located in the LSC (eight protein-coding and four tRNAs), four in the IR region (two protein-coding and two tRNAs) and one in the SSC ( $n d h A)$. One gene, the rp12 gene, was inversely linked with the duplicated 3' end exon located in the IR region and the 5' end exon located in the LSC region. These results indicated that the cp genome structure, the gene number, and the gene order of the six almond species were highly conserved and had similar roles in other genera, such as Fritillaria ${ }^{32}$, Gynostemma ${ }^{12}$ Rehmannia $^{13}$ and Aconitum ${ }^{38}$.

Comparison of border regions and sequence identity. The IR regions of the six cp genomes ranged from 26,358 bp (P. pedunculata) to 26,386 bp (P. triloba), among which $r p s 19, y c f 1, n d h F, r p l 2$, and trnH were located at the junction of the LSC/IR and SSC/IR borders. In the expansion and contraction of the IR regions, considerable changes were observed (Fig. 2). For the LSC/IR borders, the IRa/SSC boundary in the almond cp genomes contained some obvious differences (Fig. 2). The gene rps19 in the LSC of the almond species extended 


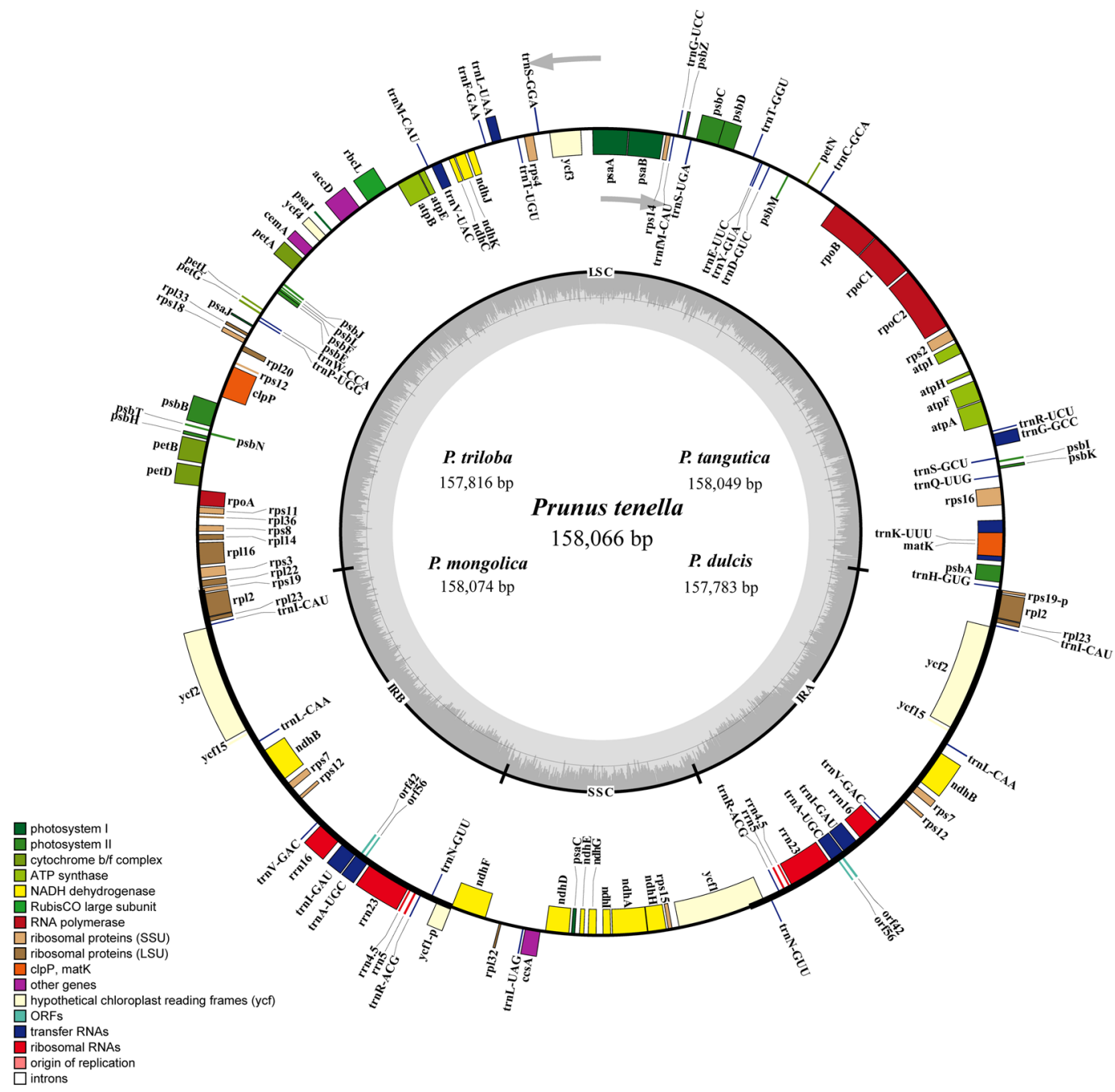

Figure 1. Gene map of the five almond chloroplast genomes. Genes drawn inside the circle are transcribed clockwise, while genes outside the circle are transcribed counterclockwise. The thick lines indicate the extent of the inverted repeats (IRa and IRb) that separate the genomes into large single-copy (LSC) and small single-copy (SSC) regions. The gene functional groups are color-coded.

into the IRa region, with a length from 179 to $187 \mathrm{bp}$. A truncated $y c f 1$ pseudogene of $984 \mathrm{bp}$ or $1161 \mathrm{bp}$ in size extended 2 bp- 12 bp into the SSC region, and overlapped with $n d h F$ by $12 \mathrm{bp}-22 \mathrm{bp}$. The gene $\operatorname{trn} H$ in the LSC region contracted 31-103 bp from the binding region of the IRb/LSC. The length of these six regions and the whole cp genome sequence were affected by the changes in the marginal regions of the IR and the SSC.

To indicate the degree of the genome divergence, the sequence identities among the almonds cpDNAs were drawn by mVISTA, with $P$. pedunculata as a reference. The whole sequences showed a high degree of similarity, with only a few areas of less than $90 \%$ similarity, indicating that the almonds' plastomes were quite conserved (Fig. 3). However, a significant divergence was mainly found in the CNS region. As expected, the coding regions had less divergence than the noncoding regions, and the IRs regions were more conservative than the single-copy regions. Similar results have been found in the chloroplast genomes of other genera, such as Gynostemma ${ }^{12}$, Rehmannia ${ }^{13}$, $_{\text {Fitillaria }}^{32}$ and Aconitum $^{38}$.

Repeat sequence analysis. Repeat motifs play an important part in phylogenetic studies and have important application value in genome rearrangement analysis. In the chloroplast genome of $P$. dulcis, 53 pairs of repeats (30 bp or longer), including 23 palindromic repeats, 17 forward repeats, six reverse repeats, and three complement repeats, were obtained using REPuter (Fig. 4A). In these repeats, one repeat is $53 \mathrm{bp}$ long, two repeats are $44 \mathrm{bp}$ long, one repeat is $43 \mathrm{bp}$ long and 44 are $30-40 \mathrm{bp}$ long (Fig. 4B). Most of these repeats (69.8\%) are located in noncoding regions, whereas some are found in genes such as $p s a B, n d h B, y c f 1, y c f 2$ and $y c f 3$ (Table S3). For more information on the six almond species repeat motifs, see Supplementary Table S3.

Repeat analysis and simple sequence repeats (SSR). Repeat sequences show high repeatability, high variability, and codominant inheritance in heterozygotes, and are thus effective molecular genetic markers in 


\begin{tabular}{|c|c|c|}
\hline Category & Gene group & Gene name \\
\hline \multirow[t]{8}{*}{ Photosynthesis related genes } & Rubisco & $r b c L$ \\
\hline & Photosystem I & psaA, psaB, psaC, psaI, psaJ \\
\hline & $\begin{array}{l}\text { Assembly/stability of } \\
\text { photosystem I }\end{array}$ & $y c f 3(\times 2)$ \\
\hline & Photosystem II & $\begin{array}{l}\text { psbA psbB, psbC, psbD, psbE, psbF, psbH psbI,psbJ, psbK, psbL psbM, psbN, } \\
p s b T, p s b Z\end{array}$ \\
\hline & ATP synthase & atpA, atpB, atpE, ${ }^{a}$ atpF, atpH, atpI \\
\hline & Cytochrome b/f complex & petA, ${ }^{a}$ petB, ${ }^{a}$ pet $D$, pet $G$, petL, petN \\
\hline & Cytochrome c synthesis & $\operatorname{ccs} A$ \\
\hline & NADPH dehydrogenase & ${ }^{a} n d h A,{ }^{a} n d h B(\times 2), n d h C, n d h D, n d h E, n d h F, n d h G, n d h H, n d h I, n d h J, n d h K$ \\
\hline \multirow[t]{2}{*}{$\begin{array}{l}\text { Transcription and translation } \\
\text { related genes }\end{array}$} & Transcription & rpoA, rpoB, ${ }^{a} r p o C 1, r p o C 2$ \\
\hline & Ribosomal proteins & $\begin{array}{l}r p s 2, r p s 3, r p s 4, r p s 7(\times 2), r p s 8, r p s 11,{ }^{c} r p s 12(\times 2), r p s 14, r p s 15,{ }^{a}{ }^{r} p s 16, \\
r p s 18, r p s 19,{ }^{a} r p l 2(\times 2), r p l 14,{ }^{a} r p l 16, r p l 20, r p l 22, r p l 23(\times 2), r p l 32, r p l 33 \\
r p l 36\end{array}$ \\
\hline \multirow[t]{2}{*}{ RNA genes } & Ribosomal RNA & $r r n 5(\times 2), r r n 4.5(\times 2), r r n 16(\times 2), r r n 23(\times 2)$ \\
\hline & Transfer RNA & 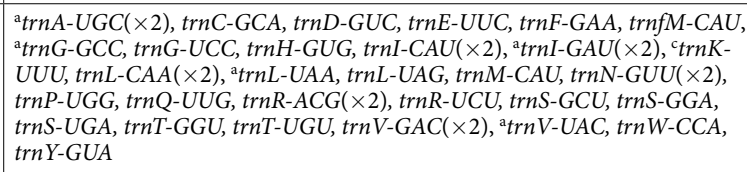 \\
\hline \multirow[t]{4}{*}{ Other genes } & RNA processing & matK \\
\hline & Carbon metabolism & cemA \\
\hline & Fatty acid synthesis & $a c c D$ \\
\hline & Proteolysis & ${ }^{b} c l p P$ \\
\hline \multirow[t]{2}{*}{ Genes of unknown function } & Conserved reading frames & $y c f 1, y c f 2(\times 2), y c f 4, y c f 15(\times 2)$ \\
\hline & $\begin{array}{l}\text { Hypothetical chloroplast } \\
\text { protein }\end{array}$ & $\operatorname{orf42}(\times 2)$, orf56 $(\times 2)$ \\
\hline Pseudogenes & & ${ }^{\Psi} y c f 1,{ }^{\Psi} r p s 19$ \\
\hline
\end{tabular}

Table 3. List of genes in the chloroplast genomes of the six almond species. ${ }^{a}$ gene containing a single intron, ${ }^{\mathrm{b}}$ gene containing two introns, ${ }^{\mathrm{c}}$ gene divided into two independent transcription units, $(\times 2)$, gene with two copies; a pseudogene is represented by ${ }^{\Psi}$.

plant species identification and in evolution and ecology studies ${ }^{12-16}$. An SSR is a repeating unit consisting of 1-6 nucleotides, also known as a short tandem repeat (STR) or a microsatellite. In this research, a large number of SSR loci (Tables S4 \& S5) were found by comparing and analyzing the genome sequences of the almond chloroplasts. Five SSR types (mononucleotide, dinucleotide, trinucleotide, tetranucleotide and pentanucleotide repeats) were obtained by comparing the six almond cp genomes; there were no hexanucleotide repeats (Table 4).

A total of 477 SSRs were detected in the almond cp genomes altogether, with each almond cp genome having 76-85 SSRs. These SSRs ranged in length from 10 to $18 \mathrm{bp}$ (Table 4 \&S5). There were 117 SSR loci in the chloroplast genomes of the six almond resources, including 96 polymorphic SSR loci. The most abundant SSR type were mononucleotide repeats (333 repeats in 84 loci), followed by dinucleotide repeats ( 91 repeats in 19 loci), tetranucleotide repeats ( 40 repeats in 8 loci), pentanucleotide repeats ( 11 repeats in 4 loci), and trinucleotide repeats ( 2 repeats in 1 loci) (Table S5). Abundant mononucleotide repeats were also found in Gynostemma ${ }^{12}$, Rehmannia ${ }^{13}$, Fritillaria $^{32}$ and Aconitum ${ }^{38}$. Therefore, mononucleotide repeats may have more influence on genetic variation than the other types of SSRs. In this research, mononucleotides almost always consisted of A/T, which was similar to previous results in Fritillaria, Rehmannia and Aconitum. These 117 SSR loci were located less frequently in the IR regions (5 SSRs) and mainly in the LSC region (100 SSRs), followed by the SSC region (12 SSRs; Table S5). Only one SSR locus extended over the IRA/SSC boundary, which was located in the protein-coding gene $y c f 1$ in the cp genome of $P$. dulcis var. ZHIPI. In addition, the SSRs in the almond cp genomes were mainly located in the intergenic spacers (IGS; 80 SSRs), followed by the introns (25 SSRs), with a minority in the coding DNA sequences (CDS; 12 SSRs). The SSR loci in the CDS regions of the almond cp genome were distributed in nine protein-coding genes (matK, rpoC2, rpoB, atpB, cemA, rps18, ndhE, ndhI, and $y c f 1$ ) (Table S5). Yu et al. ${ }^{32}$ also observed that 20 different SSRs of the Fritillaria cp genome were distributed in nine protein-coding genes ( $m a t K$, $r p o C 1, r p o C 2, c e m A, n d h D, n d h G, n d h H, y c f 2$, and $y c f 1)$, but the nine protein-coding genes were not identical. Lu et al. ${ }^{39}$ found that 15 different SSRs were located in eight protein-coding genes $(y c f 1, c e m A, r p o C 2, y c f 2, n d h H$, $r p l 22, n d h D$, and $n d h E$ ) of three Cardiocrinum chloroplast genomes. The SSR loci can be used for phylogenic study and species identification when the SSRs in the plant chloroplast genomes show abundant variation.

Recognition of highly variable regions. The highly variable region of the chloroplast genome can provide important information for phylogeny research, which can be used to identify closely related species more accurately ${ }^{32,40}$. 


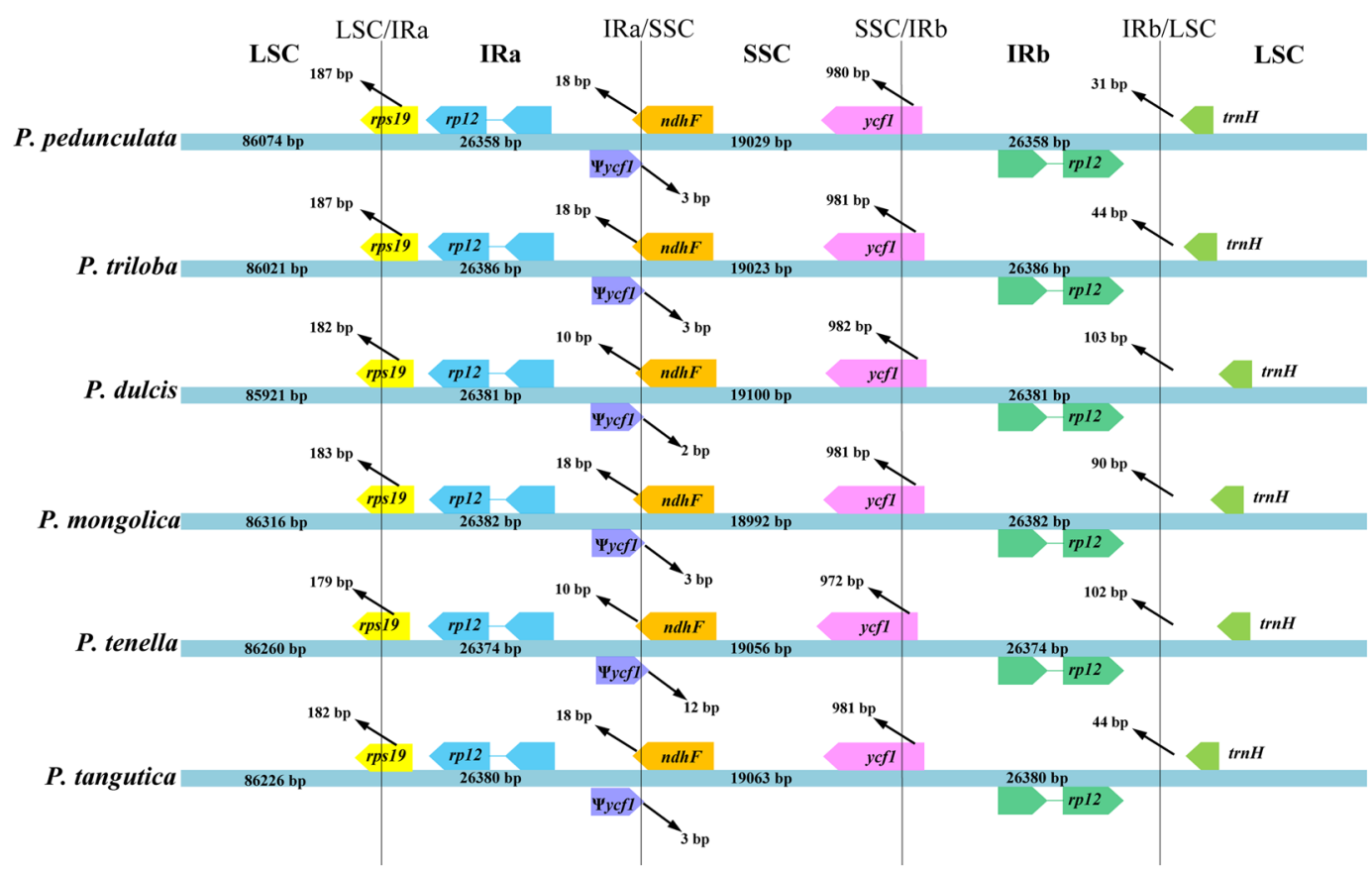

Figure 2. Comparisons of the borders of the large single-copy (LSC), small single-copy (SSC), and inverted repeat (IR) regions among the $P$. pedunculata, $P$. triloba, $P$. mongolica, . tenella, $P$. tangutica, and $P$. dulcis chloroplast genomes. The colored boxes above and below the main line indicate the adjacent border genes, where yellow indicates $r p s 19$, blue indicates $r p l 2$, purple indicates $\Psi y c f 1$ ( $\Psi$ indicates a pseudogene), brown indicates $n d h F$, pink indicates $y c f 1$, green indicates $r p l 2$, and light green indicates $\operatorname{tr} H$.

As shown in Fig. 5, the sliding window in the DnaSP 5.0 software accurately located nine highly variable regions with nucleotide variability (Pi) greater than 0.008 , including $r p s 16, r p s 16-p s b K$, atpF-atpH, $r p o B$, $y c f 3-r p s 4$, rps4-ndhJ, accD-psaI and rps7-orf42 (two highly variable regions). The highest Pi value, for $r p o B$, is 0.02867 . Two of nine highly variable regions were located in the SSC region, and seven of these regions were located in the LSC region; the nucleotide diversity values in the IR regions were not higher than 0.002 , and no highly differentiated sequence was detected, indicating that these regions were highly conserved. The sequences of these highly variable regions will provide a valuable resource for research in phyletic evolution, population genetics, species identification, and breeding directions.

Phylogenetic analysis based on the cp genomes. The phylogenetic trees were constructed from four data partitions of the cp genome of 39 Rosaceae plants: the complete cp genome (Fig. 6, Figure S3 A, and Figure S4 A), the LSC (Figure S2 A, Figure S3 B, and Figure S4 B), the SSC (Figure S2 B, Figure S3 C, and Figure S4 C), and the IR (Figure S2 C, Figure S3 D, and Figure S4 D). The complete cp genome was highly conserved, and the best resolution of the phylogenetic relationship can only be obtained by using the complete cp genome sequences. Therefore, we mainly discuss the phylogenetic relationships according to Fig. 6 . All of the Rosaceae were clustered into three main high support groups (Fig. 6). The six almond species in this study were clustered into one large group, in which the six Amygdalus resources were divided into three small clades. Three species $(P$. tenella, $P$. pedunculata and $P$. triloba) and wild cherry ( $P$. tomentosa) were grouped into clade I. Clade II included two species ( $P$. mongolica and $P$. tangutica) and wild peach ( $P$. davidiana). Clade III consisted of $P$. dulcis, $P$. persica (cultivated peach) and P. kansuensis (Gansu peach). The results of the chloroplast genome cluster analysis showed that the resources of the subgenus Amygdalus were clearly classified. Similar results were obtained by using RAxML-win32-100315 software (Figures S3 A) and MrBayes v3.2.4 software (Figures S4 A) for the same evolutionary analysis of the complete $\mathrm{cp}$ genomes. If the evolutionary analysis was not based on the complete chloroplast genome sequences, then no consistent evolutionary relationship could be obtained (Figure S2; Figure S3 B-D; Figure S4 B-D).

The relationships of the Rosaceae plants have been well resolved in previous studies ${ }^{41,42}$, but the status of almonds remains uncertain. The resources in clade I are clustered together, which is consistent with their high latitude geographical distribution. The results of clade II are consistent with the genome-based cluster analysis, in which common almond ( $P$. dulcis) is closely related to the cultivated peach (P. persica), and the Mongolic almond and Tangut almond is the closest to the cultivated peach in wild resources ${ }^{41,43}$. These results indicate that the common almond (P. dulcis) and Tangut almond (P. tangutica) belong to the same genus as the peach. The genome of $P$. pedunculata and $P$. triloba are polyploid, which is difficult to distinguish based on the evolutionary analysis of genome sequence and phenotype, which leads to an unclear evolutionary relationship of the wild almond resources. In this study, the cluster analysis showed that wild almond (P. tenella), longstalk almond $(P$. pedunculata) and flowering almond (P. triloba) are closely related and cluster together with wild cherry. 

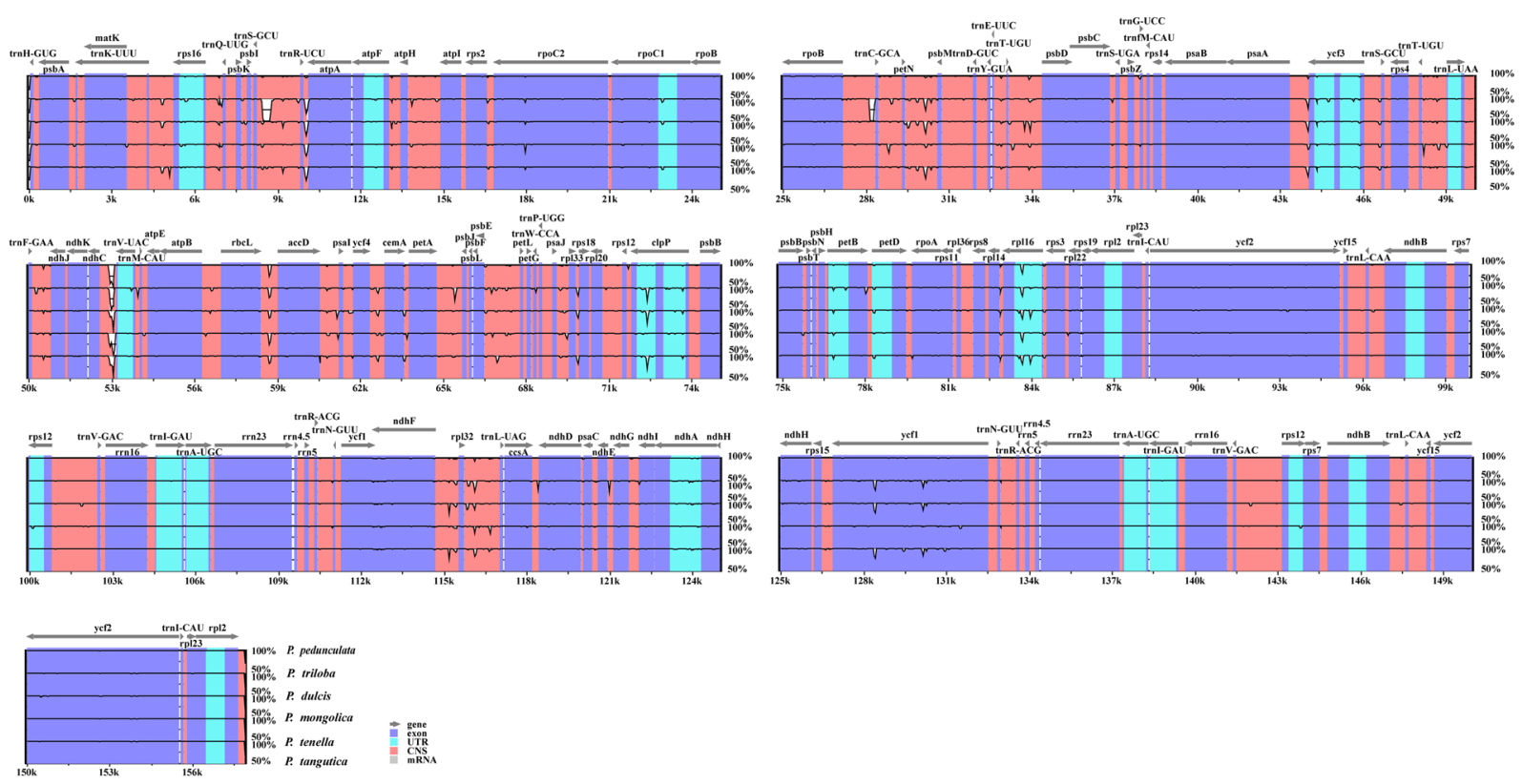

Figure 3. Visualized alignments of the six almond species cp genomes. Sequence identity plots among the five almond species chloroplast genomes were made by using mVISTA, with $P$. pedunculata as a reference. The $\mathrm{y}$-axis represents identity ranging from $50 \%$ to $100 \%$.

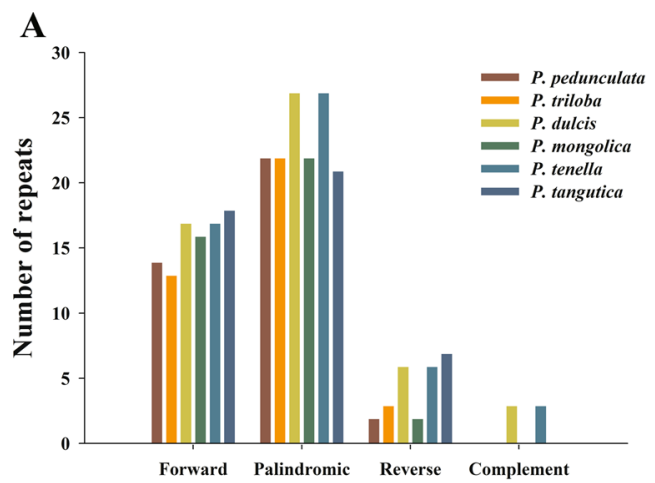

B

Repeats type

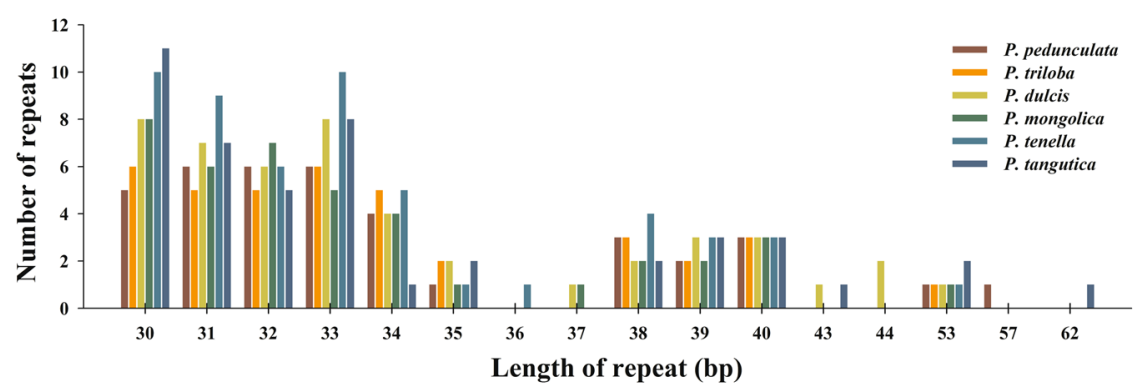

Figure 4. Analyses of repeated sequences in the six almond species chloroplast genomes. (A) Frequency of repeat types. (B) Frequency of repeat sequences by length.

Whether almond resources, including the six almond resources in this study, belong to the independent genus Amygdalus L. or the genus Prunus L. has always been a complicated matter ${ }^{2,9,10}$. Based on the phenotypic classification of classical botany, it is very difficult to clearly divide the genetic relationship of the six species. From the perspective of chloroplast clustering in this study, wild almond (P. tenella), longstalk almond (P. pedunculata) and flowering almond (P. triloba) should be classified into the genus Prunus L., while the common almond ( $P$. dulcis), Mongolic almond ( $P$. mongolica) and Tangut almond (P. tangutica) should be divided into the subgenus Amygdalus $\mathrm{L}$. 


\begin{tabular}{|l|l|l|l|l|l|l|l|l|l|l|l|l|l|l|}
\hline & $\begin{array}{l}\text { SSR } \\
\text { Soci } \\
\text { Species }\end{array}$ & $\begin{array}{l}\text { PolyM. } \\
\text { loci no. }\end{array}$ & $\begin{array}{l}\text { PolyM. } \\
\text { loci } \\
(\mathbf{1 0 0} \%)\end{array}$ & mono- & di- & tri- & tetra- & penta- & IGS & Intron & CDS & LSC & IR & SSC \\
\hline P. pedunculata & 80 & 60 & 75.00 & 56 & 14 & 1 & 8 & 1 & 51 & 16 & 13 & 71 & 2 & 7 \\
\hline P. triloba & 81 & 60 & 74.07 & 58 & 13 & 1 & 8 & 1 & 53 & 16 & 12 & 71 & 2 & 8 \\
\hline P. dulcis & 76 & 55 & 72.37 & 52 & 17 & 0 & 5 & 2 & 49 & 15 & 12 & 69 & 3 & 4 \\
\hline P. mongolica & 80 & 57 & 71.25 & 53 & 19 & 0 & 6 & 2 & 53 & 16 & 11 & 70 & 2 & 8 \\
\hline P. tenella & 85 & 63 & 74.12 & 63 & 13 & 0 & 7 & 2 & 56 & 16 & 13 & 73 & 4 & 8 \\
\hline P. tangutica & 75 & 52 & 69.33 & 51 & 15 & 0 & 6 & 3 & 50 & 12 & 13 & 65 & 2 & 8 \\
\hline Total Loci & 117 & 96 & 82.05 & 84 & 19 & 2 & 8 & 4 & 80 & 25 & 12 & 100 & 5 & 12 \\
\hline
\end{tabular}

Table 4. Simple sequence repeats (SSRs) in the six almond cp genomes.

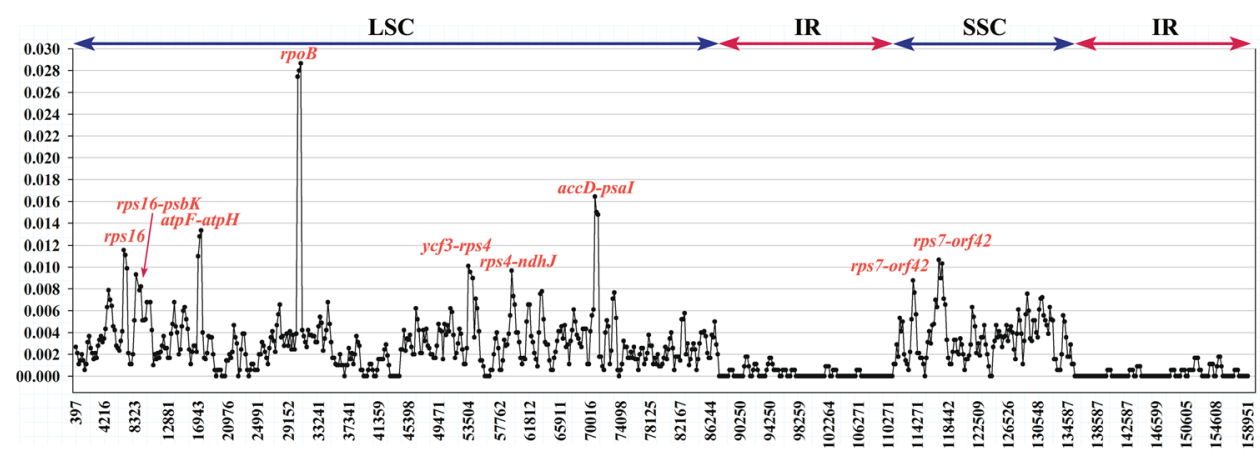

Figure 5. Sliding window analyses of the six almond species chloroplast genomes sequences. The $\mathrm{X}$-axis represents the position of the midpoint of a window, while the Y-axis represents nucleotide variability $(\mathrm{Pi})$ of each window (window length: $600 \mathrm{bp}$, step size: $200 \mathrm{bp}$ ).

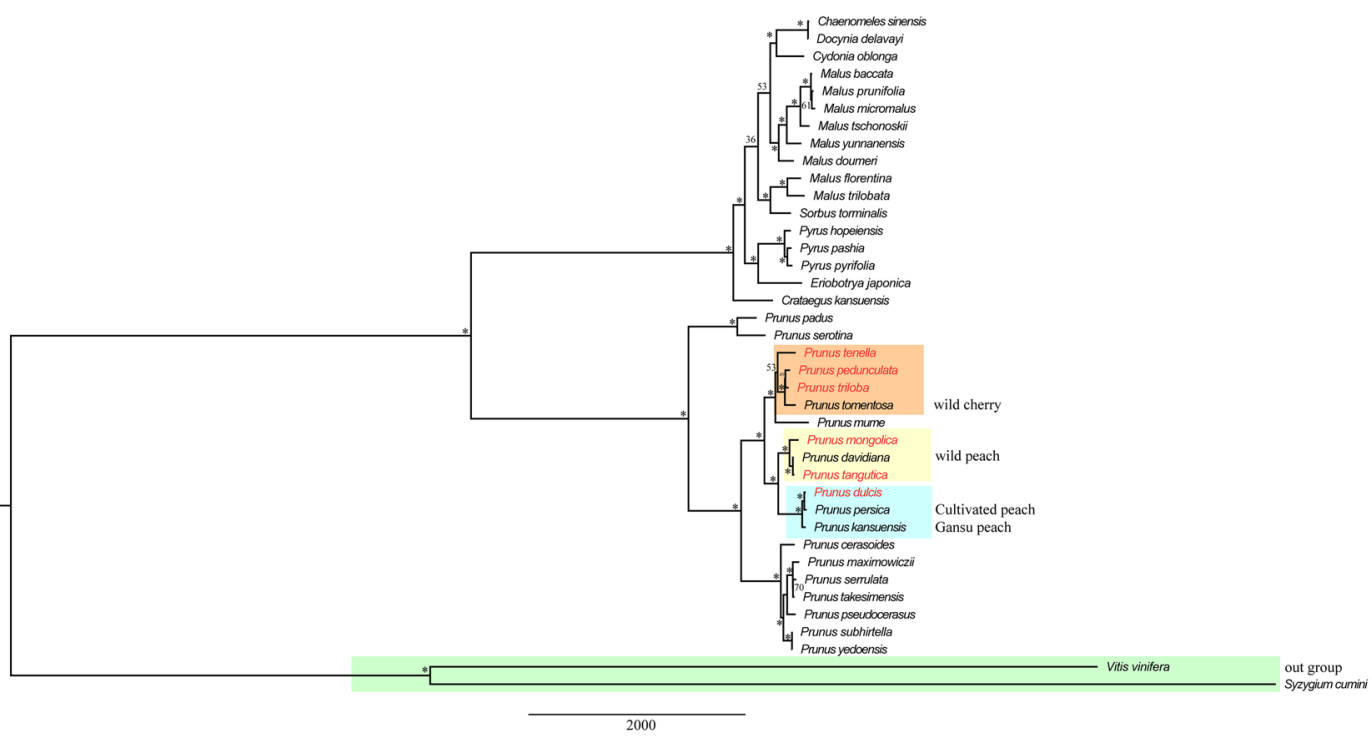

Figure 6. Phylogenetic relationships of 39 species inferred from maximum likelihood (ML) analysis based on the complete chloroplast genome sequences. The numbers above the nodes are the support values with maximum likelihood (ML) analyses, and the symbol * indicates that the support rate of the branch is $100 \%$. Clade I (orange): P. tenella, P. pedunculata, P. triloba and P. tomentosa; clade II (yellow): P. mongolica, P. tangutica and $P$. davidiana; clade III (light blue): P. dulcis, $P$. persica and P. kansuensis.

\section{Conclusions}

In this study, we report the chloroplast genomes of five almond species by de novo sequencing and compare them with one previously published almond cp genome sequence. These six complete almond cp genomes showed the most common genomic characteristics but still provided abundant genetic information for the sequence differentiation and structure research of the almond species. For repeat sequence analysis, 53 pairs of repeats (30 bp or longer) were identified. The 117 SSR loci were observed. Nine highly variable regions with nucleotide 
diversity were located. Large repeat sequences, SSRs, and highly variable regions provided possible sequence information for genetic markers. Genetic markers were used for the identification of plant germplasm resources and the improvement of plant fingerprints. A phylogenetic tree was constructed with the complete cp genomes to better understand the genetic relationships of the almond species. Three species (P. tenella, P. pedunculata and $P$. triloba) and wild cherry ( $P$. tomentosa) were group into clade I. Clade II consisted of two species ( $P$. mongolica and $P$. tangutica) and wild peach (P. davidiana). Clade III included common almond ( $P$. dulcis), cultivated peach (P. persica) and GanSu peach (P. kansuensis). Phylogenetic trees not only improve the classification of almonds but also provide guidance for selecting parents with nearer genetic relationships and hybridization compatibility in almond breeding research. These results expand the researchers' vision of almond plant diversity and promote an understanding of the evolutionary relationship among almond species. In brief, this study provides abundant resources for the study of the chloroplast genome of almonds, and has important reference value for studies on the evolution and species identification of almonds.

Received: 12 December 2019; Accepted: 12 May 2020;

Published online: 23 June 2020

\section{References}

1. Shahbandeh, M. Almond production worldwide 2018/19, by country. https://www.statista.com/statistics/632865/almondproduction-worldwide-by-country (2019).

2. Gorttapeh, A. H., Hasani, M. H. \& Ranji, H. Recognition and ecological investigation of almond species Amygdalus spp in West Azerbaijan Province. Acta Hort. 726, 253-257 (2006).

3. Ma, R. et al. Molecular analysis of almond germplasm in China. Options Mediterr. 63, 281-290 (2005).

4. Tahan, O. et al. Assessment of genetic diversity and population structure of Chinese wild almond, Amygdalus nana, using EST- and genomic SSRs. Biochem. Syst. Ecol. 37, 146-153 (2009).

5. Wang, W., Wang, H. L., Xiao, X. Z. \& Xu, X. Q. Wild almond (Amygdalus pedunculata Pall.) as potential nutritional resource for the future: studies on its chemical composition and nutritional value. J. Food Meas. Charact. 13, 250-258 (2018).

6. Wang, W., Wang, H. L., Xiao, X. Z. \& Xu, X. Q. Characterization of the complete chloroplast genome of longstalk almond (Prunus pedunculata (Pall.) Maxim.), an important sand-fixation shrub plant endemic to northern China. Conserv. Genet. Resour. 11, 419-421 (2019)

7. Wang, W., Wang, H. L., Xiao, X. Z. \& Xu, X. Q. Chemical composition analysis of seed oil from five wild almond species in China as potential edible oil resource for the future. S. Afr. J. Bot. 121, 274-281 (2019).

8. Sorkheh, K. et al. Phenotypic diversity within native Iranian almond (Prunus spp.) species and their breeding potential. Genet. Resour. Crop. Evol. 56, 947-961 (2009).

9. Browicz, K. \& Zohary, D. The genus Amygdalus L. (Rosaceae): Species relationships, distribution and evolution under domestication. Genet. Resour. Crop. Ev. 43, 229-247 (2004).

10. Esfahlan, A. J. \& Jamei, R. Properties of biological activity of ten wild almond (Prunus amygdalus L.) species. Turk. J. Biol. 36, 201-209 (2012)

11. Sorkheh, K., Dehkordi, M. K., Ercisli, S., Hegedus, A. \& Halasz, J. Comparison of traditional and new generation DNA markers declares high genetic diversity and differentiated population structure of wild almond species. Sci. Rep. 7, 5966 (2017).

12. Zhang, X. et al. Completion of Eight Gynostemma BL. (Cucurbitaceae) Chloroplast Genomes: Characterization, Comparative Analysis, and Phylogenetic Relationships. Front. Plant. Sci. 8, 1583 (2017).

13. Zeng, S. et al. The Complete Chloroplast Genome Sequences of Six Rehmannia Species. Genes 8, E103 (2017)

14. Yang, Z. \& Ji, Y. Comparative and Phylogenetic Analyses of the Complete Chloroplast Genomes of Three Arcto-Tertiary Relicts: Camptotheca acuminata, Davidia involucrata, and Nyssa sinensis. Front. Plant. Sci. 8, 1536 (2017).

15. Xu, X., Wen, J., Wang, W. \& Zheng, W. The complete chloroplast genome of the threatened Prunus cerasoides, a rare winter blooming cherry in the Himalayan region. Conserv. Genet. Resour. 10, 499-502 (2017).

16. McPherson, H. et al. Capturing chloroplast variation for molecular ecology studies: a simple next generation sequencing approach applied to a rainforest tree. BMC Ecol. 13, 8 (2013).

17. Borgström, E., Lundin, S. \& Lundeberg, J. Large Scale Library Generation for High Throughput Sequencing. PLoS One 6, e19119 (2011).

18. Cronn, R. et al. Multiplex sequencing of plant chloroplast genomes using Solexa sequencing-by-synthesis technology. Nucleic. Acids. Res. 36, e122 (2008).

19. Luo, R. et al. SOAPdenovo2: an empirically improved memory-efficient short-read de novo assembler. GigaScience 1, 18 (2015).

20. Wyman, S. K., Jansen, R. K. \& Boore, J. L. Automatic annotation of organellar genomes with DOGMA. Bioinformatics 20, 3252-3255 (2004).

21. Altschul, S. F., Gish, W., Miller, W., Myers, E. W. \& Lipman, D. J. Basic Local Alignment Search Tool. J. Mol. Biol. 215, 403-410 (1990).

22. Ashburner, M. et al. Gene Ontology: tool for the unification of biology. Nat. Genet. 25, 25-29 (2000).

23. Magrane, M. \& Consortium, U. UniProt Knowledgebase: a hub of integrated protein data. Database 2011, 1-13 (2011).

24. Tatusov, R. L. The COG database: an updated version includes eukaryotes. BMC Bioinformatics 4, 41 (2003).

25. Tatusov, R. L., Koonin, E. V. \& Lipman, D. J. A Genomic Perspective on Protein Families. Science 278, 631-637 (1997).

26. Kanehisa, M. et al. From genomics to chemical genomics: new developments in KEGG. Nucleic. Acids Res. 1, D354-D357 (2006).

27. Kanehisa, M. A database for post-genome analysis. Trends Genet. 13, 375-376 (1997).

28. Kanehisa, M., Goto, S., Kawashima, S., Okuno, Y. \& Hattori, M. The KEGG resource for deciphering the genome. Nucleic. Acids Res. 32, D277-D280 (2004).

29. Lohse, M., Drechsel, O. \& Bock, R. OrganellarGenomeDRAW (OGDRAW): a tool for the easy generation of high-quality custom graphical maps of plastid and mitochondrial genomes. Curr. Genet. 52, 267-274 (2007).

30. Kurtz, S. \& Schleiermacher, C. REPuter: fast computation of maximal repeats in complete genomes. Bioinformatics 15, 426-427 (1999).

31. Thiel, T., Michalek, W., Varshney, R. \& Graner, A. Exploiting EST databases for the development and characterization of genederived SSR-markers in barley (Hordeum vulgare L.). Theor. Appl. Genet. 106, 411-422 (2003).

32. Yu, B. et al. Chloroplast genomic resources for phylogeny and DNA barcoding: a case study on Fritillaria. Sci. Rep. 8, 1184 (2018).

33. Swofford, D. PAUP*: phylogenetic analysis using parsimony (*and other methods), version $4.0 \mathrm{~b} 10$. Sunderland, Massachusetts: Sinauer Associates (2002).

34. Stamatakis, A. RAxML version 8: a tool for phylogenetic analysis and post-analysis of large phylogenies. Bioinformatics $\mathbf{3 0}$, 1312-1313 (2014).

35. Ronquist, F. \& Huelsenbeck, J. P. MrBayes 3: Bayesian phylogenetic inference under mixed models. Bioinformatics 19, 1572-1574 (2003). 
36. Katoh, K., Kuma, K., Toh, H. \& Miyata, T. MAFFT version 5: improvement in accuracy of multiple sequence alignment. Nucleic. Acids. Res. 33, 511-518 (2005).

37. Tao, X., Ma, L., Zhang, Z., Liu, W. \& Liu, Z. Characterization of the complete chloroplast genome of alfalfa (Medicago sativa) (Leguminosae). Gene Reports 6, 67-73 (2017).

38. Park, I. et al. The complete chloroplast genome sequence of Aconitum coreanum and Aconitum carmichaelii and comparative analysis with other Aconitum species. PLoS One 12, e0184257 (2017).

39. Lu, R. S., Li, P. \& Qiu, Y. X. The Complete Chloroplast Genomes of Three Cardiocrinum (Liliaceae) Species: Comparative Genomic and Phylogenetic Analyses. Front. Plant Sci. 7, 2054 (2017).

40. Liu, H. et al. Comparative Analysis of Complete Chloroplast Genomes of Anemoclema, Anemone, Pulsatilla, and Hepatica Revealing Structural Variations Among Genera in Tribe Anemoneae (Ranunculaceae). Front. Plant Sci. 9, 1097 (2018).

41. Xiang, Y. et al. Evolution of Rosaceae Fruit Types Based on Nuclear Phylogeny in the Context of Geological Times and Genome Duplication. Mol. Biol. Evol. 34, 262-281 (2017).

42. Alioto, T. et al. Transposons played a major role in the diversification between the closely related almond (Prunus dulcis) and peach (P. persica) genomes: Results from the almond genome sequence. Plant J. 101, 455-472 (2020).

43. Yu, Y. et al. Genome re-sequencing reveals the evolutionary history of peach fruit edibility. Nat. Commun. 9, 5404 (2018).

\section{Acknowledgements}

This work was supported by the Fundamental Research Funds for the Central Non-profit Research Institution of CAF (CAFYBB2017ZA004-7), the National Natural Science Foundation of China (41501059).

\section{Author contributions}

Wei W. and Xin-Qiao X. conceived and designed the project. Xin-Qiao X. supervised the project. Wei W., Tao Y. Hui-Ling W. and Zhen-Jian L. performed the experiments. Wei W. Shang S. and Jian-Wei N. analysed the data. Wei W. prepared a draft of the manuscript and figures. All authors edited and approved the final version of the manuscript for submission.

\section{Competing interests}

The authors declare no competing interests.

\section{Additional information}

Supplementary information is available for this paper at https://doi.org/10.1038/s41598-020-67264-3.

Correspondence and requests for materials should be addressed to X.-Q.X.

Reprints and permissions information is available at www.nature.com/reprints.

Publisher's note Springer Nature remains neutral with regard to jurisdictional claims in published maps and institutional affiliations.

(c) (i) Open Access This article is licensed under a Creative Commons Attribution 4.0 International License, which permits use, sharing, adaptation, distribution and reproduction in any medium or format, as long as you give appropriate credit to the original author(s) and the source, provide a link to the Creative Commons license, and indicate if changes were made. The images or other third party material in this article are included in the article's Creative Commons license, unless indicated otherwise in a credit line to the material. If material is not included in the article's Creative Commons license and your intended use is not permitted by statutory regulation or exceeds the permitted use, you will need to obtain permission directly from the copyright holder. To view a copy of this license, visit http://creativecommons.org/licenses/by/4.0/.

(c) The Author(s) 2020 